\title{
Tumor-Infiltrating Leukocyte Compositions and Prognostic Immune-Related Genes in Pancreatic Cancer.
}

\section{Buwei Teng}

Department of General Surgery, Shanghai General Hospital of Nanjing Medical University

\section{Yuhan Yang}

Department of General Surgery, Shanghai General Hospital, Shanghai Jiao Tong University School of Medicine

\section{Zengya Guo}

Department of General Surgery, Shanghai General Hospital, Shanghai Jiao Tong University School of Medicine

\section{Kundong Zhang}

Department of General Surgery, Shanghai General Hospital, Shanghai Jiao Tong University School of Medicine

\section{Xiaofeng Wang}

Department of General Surgery, Shanghai General Hospital, Shanghai Jiao Tong University School of Medicine

\section{Weiwei Chen}

Department of General Surgery, Shanghai General Hospital, Shanghai Jiao Tong University School of Medicine

\section{Zhengjun Qiu ( $\nabla$ qiuzjdoctor@sina.com )}

Department of General Surgery, Shanghai General Hospital of Nanjing Medical University

\section{Research Article}

Keywords: pancreatic cancer, immunogenomic landscape, The Cancer Genome Atlas, prognostic index

Posted Date: December 28th, 2020

DOI: https://doi.org/10.21203/rs.3.rs-119677/v1

License: (1) This work is licensed under a Creative Commons Attribution 4.0 International License. Read Full License 


\section{Abstract}

Background:Pancreatic cancer (PC) is one of the most common cancers $₫$ which has poor prognosis.At present, abundant genetic PC samples can be obtained from The Cancer Genome Atlas (TCGA) database to finish comprehensive and reliable immunogenomic analysis. Thus, there is an urgent need to systematically explore the immunogenome of PC to obtain good prognosis.

Methods: In this study, according to TCGA and The Genotype-Tissue Expression (GTEx) databases, we investigated the different compositions of leukocytes between PC and normal pancreas tissues, and analyzed the expressions of immune-related genes (IRGs) and the overall survival (OS) of 178 PC patients. Subsequently, computational difference algorithm and COX regression analyses were employed to assess the differentially expressed and OS-related IRGs in PC patients. Moreover, the underlying action mechanisms and properties of these IRGs were investigated by using computational biology. Finally, multivariable COX analysis was used to develop a novel prognostic biomarker for PC according to these IRGs.

Results:The results showed that $\mathrm{CD} 4^{+}$memory $\mathrm{T}$ cells and $\mathrm{M} 0$ macrophages were more common and highly dominated in PC tissues relative to the non-tumor tissues. Functional enrichment analysis demonstrated that the differentially expressed and OS-related IRGs were actively involved in the PI3K-Akt signaling pathway. A prognostic signature according to these differentially expressed IRGs (CD2AP, IL20RB, MYEOV, NUSAP1, PCDH1, RAB27B, TNFSF10, TOP2A, TPX2, TYK2, WNT7A and BUB1B) was moderately used for prognostic predictions. Further study indicated that RAB27B was negatively related to CD4 T cells while TYK2 was positively correlated with CD4 T cells.

Conclusions: Taken together, this study screened several significant IRGs, demonstrated the drivers of immune repertoire, and indicated the importance of these PC-specific IRGs in the prognosis of PC.

\section{Background}

Pancreatic cancer (PC), one of the most common cancers, has been becoming the second factor to cause the cancer-related deaths in America[1]. Even if various therapies have been applied on the treatment of $\mathrm{PC}$, including surgical resection and adjuvant treatments such as chemotherapy, the 5-year overall survival (OS) rate does not exceed $5 \%$ and the average survival time is only approximately 3 to 6 months [2]. A previous study indicated that the high mortality of PC patients was primarily resulted from difficulty in early diagnosis, aggressive local invasion and early metastasis [3]. Thus, it is crucial to find novel diagnostic biomarkers for $\mathrm{PC}$ and understand its potential action mechanism.

At present, immunotherapy has become an important means of targeted therapy for tumors, which can attack tumors through activating the immune system[4]. However, many immunotherapies with significant efficacies in other cancers have poor efficacy in the treatment for PC [5]. One of the characteristics of PC tumor microenvironment in mice and humans is that there are a large numbers of non-tumor components in tumors, collectively called as matrix [6]. In PC, matrix accounts for $50 \%$ of the 
total mass of tumors, and was proved to suppress spontaneous and therapeutically induce anti-tumor immunity [7]. In fact, a widespread immune strategy limits the efficacy of immunotherapy, and proposes that the main escape mechanism used by cancer cells is that to sensitize PC to myelopoiesis state through secreting metabolites, cytokines, immune factors and chemokines, thereby altering preclinical and clinical outcomes.

Tumor-associated macrophages (TAM) account for $15-20 \%$ of the total numbers of immune cells. In general, circulating blood monocytes firstly migrate into tissues, and are subsequently differentiated into resident tissue macrophages [8]. Affected by some environmental factors including microbial products and cytokines, these resident macrophages can be activated, and the activated macrophages are usually divided into classical activated (M1) and alternatively activated (M2) macrophages [9]. M1 macrophages can induce T-helper-1 (Th1) cell responses. On the contrary, M2 macrophages can exhibit different phenotypes depending on the induced signals including IL-4, IL-13, IL-10 and glucocorticoid hormones [10]. In most tumors, the phenotype of macrophages is consistent with that at the decomposition stage of wound healing, but TAM often exhibit immunosuppressive phenotype. In addition, the phenotypic analysis for TAM transcriptome showed that TAM expressed some immunosuppressive genes including arginase I, IL-10, TGF-beta and so on [11].

Activation of effector T cells is a key part to compose the immunotherapy for PC. Cytotoxic CD8 + effector T cells are the core of successful tumor immune responses, and the tumor infiltration of CD8 + T cells was proved to be related to the prognosis of PDAC patients [12]. Whereas, in most PC patients and mice, $C D 8+T$ cells were very rare and their activated levels obviously reduced, indicating that the infiltration and/or activity of tumor-responsive $T$ cells are suppressed[13].

Therefore, this study aimed to gain insights into the potential clinical applications of some immunerelated genes (IRGs) on prognosis and their potentials as the targeted biomarkers for PC therapy. At first, we integrated IRG expressions with clinical data to assess the OS of PC patients using computational methods. Subsequently, we systematically analyzed the expressions of IRGs, and screened independently prognostic biomarkers for PC patients. Finally, bioinformatics analysis was performed to investigate the potential action mechanism. This study lays a foundation for early diagnosis and individualized immunotherapy of PC.

\section{Methods}

\section{Data source and preprocessing}

The original mRNA expressions and corresponding clinical data of PC patients were obtained from TCGA database (https://tcga-data. nci.nih.gov/tcga/). The samples without enough clinical or/and survival data were eliminated. In addition, the sex and stage data from the American Joint Committee on Cancer (AJCC) were available. Considering that there were only 4 normal pancreas tissues in TCGA database, we 
got the mRNA expression data of normal pancreas from The Genotype-Tissue Expression (GTEx) database established by the NIH Common Fund.

|log2 fold changel $\geq 2$ and $\mathrm{P}<0.05$ were used as the criteria to screen DEG. Then, according to the "limma" R package available at http://bioconductor.org/packages/release/bioc/html/limma.html, the DEG in tissues were selected for further analyses. The volcano map and heatmap were drawn using "ggplwot2" originated from R platform (version 3.5.2).

\section{The establishment of risk-score model}

Next, we conducted normalized and differential analyses. 224 IRGs with significant differences were selected using the univariate Cox model to perform the least absolute shrinkage and selection operator (LASSO) analysis, thereby screening the IRGs highly related to OS. Following data processing, coefficients ( $\beta i)$ were calculated with 12 selected key IRGs using the multivariate Cox model. Then, the score model consisted of $\beta i$ and IRG mRNA expressions was established as follows: risk score $=\sum 5$ ( $\beta i$ * Expi). In this formula, the $\mathrm{i}=1$ risk score for each subject was calculated. Using the mean risk score as cutoff value, all eligible subjects were assigned into high- and low-risk groups. Finally, the prognosis differences between high- and low-risk patients were presented by using Kaplan-Meier survival curves.

\section{Functional enrichment analysis}

The "Metascape" and "clusterProfiler" web tools were used to conduct gene ontology consortium and Kyoto Encyclopedia of Genes and Genomes (KEGG) pathway analyses to investigate the biological processes and potential pathways that IRGs were involved.

\section{Compositions of immune cells and their correlations with IRGs}

The CIBERSORT algorithm is a tool for estimating the abundances of immune cells using transcriptomic data. This software uses liner support vector regression (SVR) to deconvolve the fractions of immune cells from the transcriptional profiles of a bulk tumor samples based on the signature matrix. The correlations between IRGs and immune cells were established by Tumor Immune Estimation Resource (TIMER).

\section{Results}

\section{Compositions of immune cells in PC}

To systematically investigate the different immune cell compositions between PC and non-tumor tissues, CIBERSORT which is an analytical tool to provide an estimation of the abundances of member cell types in a mixed cell population, using gene expression data,was employed to calculate the proportion of each kind of immune cells. 
As Figs. $1 \mathrm{a}$ and $1 \mathrm{~b}$ shown, resting $\mathrm{CD} 4^{+}$memory $\mathrm{T}$ cells, $\mathrm{M} 0$ and $\mathrm{M} 2$ macrophages, $\mathrm{T}$ cell $\mathrm{CD} 8$ and $\mathrm{B}$ cells were the 5 most common immune cells.

Compared with non-tumor pancreas tissues $(n=21)$, resting memory CD $4^{+} T$ cells, activated CD4 memory $\mathrm{T}$ cells, regulatory $\mathrm{T}$ cells, M0 macrophages, resting dendritic cells and activated dendritic cells were highly dominated $(P<0.001)$ in PC tissues $(n=178)$. To contrast, follicular helper T cells, monocytes and M2 macrophages predominated $(P<0.001)$ in the non-tumor tissues relative to PC tissues (Fig. 1c).

\section{The identification of differentially expressed genes (DEG)}

As shown in Figs. 2a and 2b, a total of 4,846 DEG were identified by using edgeR algorithm, of which 2,558 were up-regulated and 2288 were down-regulated.

Subsequently, 1,206 differentially expressed IRGs were selected for further analyses

(Fig. 2c). The functional enrichment analysis showed that the inflammation-related pathway was most enriched, and "leukocyte migration", "T cell activation" and "positive regulation of cell adhesion" were the most frequent biological terms (Fig. 2d). In addition, KEGG analysis demonstrated that cytokine-cytokine receptor and PI3K-Akt signaling pathways were most enriched by these IRGs (Fig. 2e).

\section{The identification of OS-related IRGs}

To investigate the association of IRG expressions with the OS of patients, 224 IRGs with significant differences $(P<0.05)$ were selected and univariate regression analysis was employed. Consistent with the results from the functional enrichment analysis for DEG, the OS-related IRGs were also most enriched in the gene ontology terms related to cell interaction and movement. In addition, the PI3K-Akt pathway was also most enriched as demonstrated by KEGG analysis (Fig. 3).

\section{The identification of hub IRGs}

To identify the IRGs that are actively involved in the occurrence and prognosis of $P C$, the differentially expressed IRGs markedly related to clinical outcomes $(P<0.05)$ were selected for further analyses. The protein-protein interaction (PPI) network analysis showed that CDK1, BUB1 and AURKB were the 3 hub genes (Figs. 4a and 4b). And functional enrichment analysis demonstrated that these 3 hub genes actively participated in PI3K-Akt pathway (Fig. 4c).

\section{Assessment of clinical outcomes}

As above mentioned, 224 IRGs with significant differences $(P<0.05)$ were selected using the univariate Cox model to perform the LASSO analysis, thereby screening the IRGs highly related to OS. LASSO analysis is helpful to select variables and regularize when fitting the generalized linear model, and can avoid over-fitting to some extent. In addition, since the complexity of LASSO analysis is depended on coefficient $(\mathrm{Y})$, a model with less variables can be obtained through conducting a penalty ratio according to their sizes. At last, a relatively small numbers of closely related indicators were obtained to draw the diagram with the partial possibility deviance vs $\log (\mathrm{Y})$. 
According to the multivariate Cox and LASSO regression analyses, we established a prognostic signature to divide the PC patients into 2 groups that had discrete clinical outcomes about OS (Figs. 5a-5b). The formula was as follows:

CD2AP expression * $1.73856+$ IL20RB expression * 0.37556 + MYEOV expression * $0.59251+$ NUSAP1 expression * $2.94630+\mathrm{PCDH} 1$ expression * $(-2.52506)+\mathrm{RAB} 27 \mathrm{~B}$ expression * $1.03662+\mathrm{TNFSF} 10$ expression * 1.65240 + TOP2A expression * (-1.55616) + TPX2 expression * $2.15252+$ TYK2 expression * $(-3.71478)+$ WNT7A expression * $0.52738+$ BUB1B expression * $(-2.27612)$

The immune-related prognostic index is a key tool to distinguish among PC patients according to the potential discrete clinical outcomes (Fig. 5c-5e). The area value under receiver operating characteristic curve was 0.802 , demonstrating the moderate prognostic potential of IRGs for OS (Fig. 5f).

\section{Correlations between the OS-related IRGs and immune cells}

Next, the relationships between the OS of PC patients and the expressions of 12 IRGs was assessed by using Kaplan-Meier analysis (Figs. 6).

Based on the TIMER, a web server for comprehensive analysis of tumor-infiltrating immune cells, we further analyzed the correlations between expressions of the 12 IRGs and immune infiltrates. As shown in Figs. 7a and 7b, RAB27B was negatively related to CD4 T cells while TYK2 was positively related to CD4 T cells.

\section{Discussion}

Although the immunotherapy for tumors has exhibited good effects on many cancers including melanoma and small cell lung cancer. However, it is not sensitive to most PC patients to date. Therefore, it is necessary to systematically study the tumor-infiltrating immune cells and immune genes in PC, which will reveal their clinical significances and relationships. In fact, the 5-year survival rate of PC is extremely poor, and the study on immune genes reveals their possible relationships with clinical prognosis, which is contributed to evaluate the OS of PC. In this present study, we obtained the DEG between tumor and nontumor tissues and studied their interactions. And systematical bioinformatics analysis further explored their molecular mechanisms. Most importantly, according to the screened and differentially expressed IRGs, a separate post-processing feature was proposed to determine immune cell infiltrations and evaluate the potential clinical outcomes of PC patients.

Our analysis for the immune cell compositions according to TCGA and GTEx databases showed that resting CD4 + memory $\mathrm{T}$ cells, $\mathrm{M} 0$ and $\mathrm{M} 2$ macrophages, CD8 T cells and $\mathrm{B}$ cells were the main immune cells. Previous studies have shown that PC is described as a cold tumor in the past [14], and the TAM are 
the most important immune cells in PC tissues, accounting for 15-20\% of immune cells [15]. Many other evidences indicated that the tumor-related macrophages were mainly M2 macrophages [16], which could induce the epithelial-mesenchymal transition (EMT) of PC [17]. Meanwhile, these M2 macrophages could also secret some factors including CCL22 and IL-10, thereby suppressing the functions of CD $4+$ and CD 8 $+T$ cells [18-19].In addition, the macrophage-derived exosomes were proved to also induce the drug resistance in $\mathrm{PC}[20]$.

It is reported that the intra-tumor CD4 + Th2 cell infiltrates and FoxP3 + Tregs were also related to the decreased survival of PC patients [21]. In addition, the activated Tregs could induce M2-like macrophage differentiation[22], and the depletion of macrophages in KPC mouse model was proved to markedly reduce tumor metastasis and be associated with the reductions of CD $4+$ and CD25 $+T$ cell levels [23].

In summary, PC is a typical cold tumor, and its immune microenvironment is mainly infiltrated by cancerpromoting CD 4 T cells and TAM. The immunotherapy for immune checkpoints such as PD- 1 and CTLA4 mainly promotes the immunosuppression for $\mathrm{CD} 8+\mathrm{T}$ cells, and has a good effect on heat tumors including melanoma and small cell lung cancer. Our study showed that CD4 $+T$ cells were the predominant cells in PC. Therefore, the immunotherapy targeted for suppressing T cells and TAM may be more effective for PC.

Our analysis for immune genes revealed that both differentially expressed and OS-related immune genes were mainly concentrated at the PI3K-Akt signaling pathway. Interestingly, the PI3K-AKTmTOR was proved to be a key signaling pathway to induce PC and be frequently activated in PC [24].

To establish simple and convenient methods to monitor the immune status and predict clinical outcomes of PC patients, an immune-based prognostic signature was proposed with 12 hub IRGs related to OS. No studies on the function and mechanism of TNFSF10, CD2AP, PCDH1, MYEOV, NUSAP1 in PC have been reported. Among them, CD2AP was reported to induce CD 4 T cell differentiation [25], and PCDH1 can bind to SMAD3 thus suppressing the TGF- $\beta 1$-induced gene transcription [26].

TOP2A, a co-activator of $\beta$-catenin, activates PC EMT process[27]. And high expression of IL20RB, BUB1B and WNT7A have been reported to have correlation with shorter survival of PC patients [28-31].However, our analysis based on TCGA database demonstrated that high BUB1B expression was related to better prognosis. TPX2 mRNA and protein are highly expressed in PC cells or tumors, and previous studies indicated that the TPX2 knockdown with TPX2-specific RNA could effectively reduce the growth but induce apoptosis of PC cells in media, and suppressed the growth of PC in soft agar and nude mice [3233].

Among the 12 genes, TYK2 was reported to be positively related to CD4 T cells while RAB27B was negatively related to CD4 T cells [34]. TYK2 belonged to JAK family could activate STAT3 and promote the expressions of the critical genes related to the growth, survival and immunosuppression of tumors [35]. In addition, TYK2 signal was also critical for the IL-23-induced IL-17 secretion in $\gamma \delta$ T cells, which could promote the release of IL-27 in macrophages [36]. Meanwhile, IL-27 could lead to markedly 
increased Th1/Tc1, Th2/Tc2, Th17/Tc17, Th9, and Th22 cells [37]. More importantly, RAB27B was proved to be used as a potential biomarker for metastasis and prognosis of breast cancer and lung adenocarcinoma [38-39], and play significant roles in the invasion, proliferation and apoptosis of PC cells, as well as in chemotherapy resistance [40]. Our study demonstrated that RAB27B expression was also negatively related to CD $4 \mathrm{~T}$ cell infiltration. But, the function of RAB27B in suppressing CD 4 T cells needs to be further studied.

\section{Conclusions}

In conclusion, through the analysis for PC patients based on TCGA and GTEx databases, we obtained differentially expressed and OS-related core immune genes, and then established a prognostic model. In addition, we also analyzed the infiltration and clustering of immune cells in PC and their associations with the above genes.

\section{Abbreviations}

PC Pancreatic cancer

TCGA The Cancer Genome Atlas

GTEx Genotype-Tissue Expression

IRGs immune-related genes

OS overall survival

TAM Tumor-associated macrophages

Th1 T-helper-1

AJCC American Joint Committee on Cancer

SVR support vector regression

KEGG Kyoto Encyclopedia of Genes and Genomes

PPI protein-protein interaction

LASSO least absolute shrinkage and selection operator

TIMER Tumor Immune Estimation Resource

EMT epithelial-mesenchymal transition

\section{Declarations}




\section{Ethics approval and consent to participate}

Not applicable

\section{Consent for publication}

Not applicable

\section{Availability of data and materials}

The datasets used and analysed during the current study are available TCGA database (https://tcga-data. nci.nih.gov/tcga/) and The Genotype-Tissue Expression (GTEx) database freely.

\section{Competing interests}

The authors declare that they have no competing interest

\section{Funding}

This work was supported by grants from the National Natural Science Foundation of China (81372640) received by Z.Q.

\section{Authors' contributions}

$\mathrm{BT}, \mathrm{YY}$ and $\mathrm{ZQ}$ designed the analysis, analyzed the data and revised the manuscript. ZG wrote the manuscript. KZ,XW and WC prepared the figures.All of the authors discussed the results and reviewed the manuscript.

\section{Acknowledgements}

Not applicable

\section{References}

1. Anuradha R, Munisankar S, Bhootra Y, Dolla C, Kumaran P, Nutman TB and Babu S. Pancreatic cancer biology and genetics from an evolutionary perspective. Nat Rev Cancer. 2016;16:553-565.

2. Bapat AA, Hostetter G, Von Hoff DD and Han H. Perineural invasion and associated pain in pancreatic cancer. Nature reviews Cancer. 2011;11:695-707. 
3. Jin $\mathrm{H}, \mathrm{Wu} Y$, Tan $\mathrm{X}$. The role of pancreatic cancer-derived exosomes in cancer progress and their potential application as biomarkers. Clinical \& translational oncology:official publication of the Federation of Spanish Oncology Societies and of the National Cancer Institute of Mexico.2017;19:921-930.

4. Sethi V, Vitiello GA, Saxena D, Miller Gand Dudeja V. The Role of the Microbiome in Immunologic Development and its Implication For Pancreatic Cancer Immunotherapy. Gastroenterology. 2019;156:2097-2115.

5. Cheung PF, Lutz M, Siveke JT. Immunotherapy and Combination Strategies in Pancreatic Cancer: Current Status and Emerging Trends. Oncology research and treatment. 2018;41:286-290.

6. Morrison AH,Byrne KT,Vonderheide RH. Immunotherapy and Prevention of Pancreatic Cancer. Trends in cancer.2018;4: 418-428.

7. Balachandran VP, Beatty GL, Dougan SK. Broadening the Impact of Immunotherapy to Pancreatic Cancer: Challenges and Opportunities. Gastroenterology.2019;15:2056-2072.

8. Komohara Y, Fujiwara Y, Ohnishi Kand Takeya M. Tumor-associated macrophages: Potential therapeutic targets for anti-cancer therapy. Advanced drug delivery reviews.2016;99:180-185.

9. Martinez FO, Gordon S.The M1 and M2 paradigm of macrophage activation: time for reassessment.F1000prime reports.2014;6:13.

10. Cui R, Yue W, Lattime EC, Stein MN, Xu Qand Tan XL. Targeting tumor-associated macrophages to combat pancreatic cancer. Oncotarget. 2016;7:50735-50754.

11. Colvin EK. Tumor-associated macrophages contribute to tumor progression in ovarian cancer. Front Oncol.2014;4:137.

12. Bauer C, Kuhnemuth B, Duewell P, Ormanns S, Gress Tand Schnurr M. Prevailing over T cell exhaustion: New developments in the immunotherapy of pancreatic cancer. Cancer Lett. 2016;381:259-268.

13. Liu F, Saif MW. T cell optimization for the treatment of pancreatic cancer. Expert opinion on biological therapy.2017;17:1493-1501.

14. Xu JW, Wang L, Cheng YG, Zhang GY, Hu SY, Zhou B and Zhan HX. Immunotherapy for pancreatic cancer: A long and hopeful journey. Cancer Lett.2018;425:143-151.

15. Ngambenjawong C, Gustafson HH, Pun SH. Progress in tumor-associated macrophage (TAM)targeted therapeutics. Advanced drug delivery reviews.2017; 144: 206-221.

16. Nahrendorf M, Swirski FK. Abandoning M1/M2 for a Network Model of Macrophage Function. Circulation research. 2016;119:414-417.

17. Liu CY, Xu JY, Shi XY, Huang W, Ruan TY, Xie P and Ding JL. M2-polarized tumor-associated macrophages promoted epithelial-mesenchymal transition in pancreatic cancer cells, partially through TLR4/IL-10 signaling pathway. Laboratory investigation:a journal of technical methods and pathology.2013;93: 844-854. 
18. Franklin RA, Liao W, Sarkar A, Kim MV, Bivona MR, Liu K, Pamer EGand Li MO. The cellular and molecular origin of tumor-associated macrophages. Science(New York, NY).2014;344:921-925.

19. Horlad H, Fujiwara Y, Takemura K, Ohnishi K, Ikeda T, Tsukamoto H, Mizuta H, Nishimura Y, Takeya M and Komohara Y. Corosolic acid impairs tumor development and lung metastasis by inhibiting the immunosuppressive activity of myeloid-derived suppressor cells. Molecular nutrition \& food research. 2013; 57:1046-1054.

20. Binenbaum Y, Fridman E, Yaari Z, Milman N, Schroeder A, Ben David G, Shlomi T and Gil Z. Transfer of miRNA in Macrophage-Derived Exosomes Induces Drug Resistance in Pancreatic Adenocarcinoma. Cancer Res.2018;78: 5287-5299.

21. De Monte L, Reni M, Tassi E, Clavenna D, Papa I, Recalde H, Braga M, Di Carlo V, Doglioni C and Protti MP. Intratumor T helper type 2 cell infiltrate correlates with cancer-associated fibroblast thymic stromal lymphopoietin production and reduced survival in pancreatic cancer. The Journal of experimental medicine.2011;208:469-478.

22. Weirather J, Hofmann UD, Beyersdorf N, Ramos GC, Vogel B, Frey A, Ertl G, Kerkau T and Frantz S. Foxp3 + CD4 + T cells improve healing after myocardial infarction by modulating monocyte/macrophage differentiation. Circulation research.2014;115:55-67.

23. Griesmann H, Drexel C, Milosevic N, Sipos B,Rosendahl J, Gress TM and Michl P.Pharmacological macrophage inhibition decreases metastasis formation in a genetic model of pancreatic cancer. Gut.2017;66:1278-1285.

24. Ebrahimi S, Hosseini M, Shahidsales S, Maftouh M, Ferns GA, Ghayour-Mobarhan M, Hassanian SM and Avan A. Targeting the Akt/PI3K Signaling Pathway as a Potential Therapeutic Strategy for the Treatment of Pancreatic Cancer. Current medicinal chemistry.2017;24:1321-1331.

25. Raju S, Kometani K, Kurosaki T, Shaw AS and Egawa T. The adaptor molecule CD2AP in CD4 T cells modulates differentiation of follicular helper T cells during chronic LCMV infection.PLoS pathogens.2018;14:e1007053.

26. Faura Tellez G, Vandepoele K, Brouwer U, Koning H, Elderman RM, Hackett TL, Willemse BW, Holloway J, Van Roy F, Koppelman GH and Nawijn MC. Protocadherin-1 binds to SMAD3 and suppresses TGF-beta1-induced gene transcription. American journal of physiology Lung cellular and molecular physiology.2015;309:L725-735.

27. Pei YF, Yin XM, Liu XQ.TOP2A induces malignant character of pancreatic cancer through activating beta-catenin signaling pathway.Biochimica et biophysica acta Molecular basis of disease.2018;1864:197-207.

28. Dong S, Huang F, Zhang H and Chen Q. Overexpression of BUB1B, CCNA2, CDC20, and CDK1 in tumor tissues predicts poor survival in pancreatic ductal adenocarcinoma. Bioscience reports. 2019;39.

29. Haider S, Wang J, Nagano A, Desai A, Arumugam P, Dumartin L, Fitzgibbon J, Hagemann T, Marshall JF, Kocher HM, Crnogorac-Jurcevic T, Scarpa A, Lemoine NR and Chelala C. A multi-gene signature predicts outcome in patients with pancreatic ductal adenocarcinoma. Genome medicine.2014;6:105. 
30. Shindo K, Yu J, Suenaga M, Fesharakizadeh S, Cho C, Macgregor-Das A, Siddiqui A, Witmer PD, Tamura, K, Song TJ, Navarro Almario JA, Brant A, Borges M, Ford M, Barkley T, He J, Weiss MJ, Wolfgang CL, Roberts NJ, Hruban RH, Klein AP and Goggins, M. Deleterious Germline Mutations in Patients With Apparently Sporadic Pancreatic Adenocarcinoma.Journal of clinical oncology: official journal of the American Society of Clinical Oncology. 2017 ;35:3382-3390.

31. Wu DJ, Jiang YS, He RZ, Tao LY, Yang MW, Fu XL, Yang JY and Zhu K. High expression of WNT7A predicts poor prognosis and promote tumor metastasis in pancreatic ductal adenocarcinoma.Sci Rep.2018;8: 15792.

32. Ludwig R, Teran FJ, Teichgraeber $U$ and Hilger I. Nanoparticle-based hyperthermia distinctly impacts production of ROS, expression of Ki-67, TOP2A, and TPX2, and induction of apoptosis in pancreatic cancer. International journal of nanomedicine.2017;12:1009-1018.

33. Warner SL, Stephens BJ, Nwokenkwo S, Hostetter G, Sugeng A, Hidalgo M, Trent JM, Han H and Von Hoff DD. Validation of TPX2 as a potential therapeutic target in pancreatic cancer cells. Clin Cancer Res. 2009;15:6519-6528.

34. Nakamura R, Shibata K, Yamada H, Shimoda K, Nakayama Kand Yoshikai Y. Tyk2-signaling plays an important role in host defense against Escherichia coli through IL-23-induced IL-17 production by gammadelta T cells. Journal of immunology (Baltimore,Md:1950).2008;181:2071-2075.

35. Herrmann A, Lahtz C, Nagao T, Song JY, Chan WC, Lee H, Yue C, Look T, Mulfarth R, Li W, Jenkins K, Williams J, Budde LE, Forman S, Kwak L, Blankenstein T and Yu H. CTLA4 Promotes Tyk2-STAT3Dependent B-cell Oncogenicity.Cancer Res.2017;77:5118-5128.

36. Bosmann M, Strobl B, Kichler N, Rigler D, Grailer JJ, Pache F, Murray PJ, Muller M and Ward PA. Tyrosine kinase 2 promotes sepsis-associated lethality by facilitating production of interleukin27.Journal of leukocyte biology. 2014;96:123-131.

37. Anuradha R, Munisankar S, Bhootra Y, Dolla C, Kumaran P, Nutman TB and Babu S. Modulation of CD4(+) and CD8(+) T Cell Function and Cytokine Responses in Strongyloides stercoralis Infection by Interleukin-27 (IL-27) and IL-37.Infection and immunity.2017;85:e00500-17.

38. Koh HM, Song DH. Prognostic role of Rab27A and Rab27B expression in patients with non-small cell lung carcinoma. Thoracic cancer.2019;10:143-149.

39. Zhang L, Fan W, Xu L, Mao Q, ChenY,Mao Y,Xu L and Wang J. Rab27b Is a Potential Indicator for Lymph Node Metastasis and Unfavorable Prognosis in Lung Adenocarcinoma.Disease markers. 2018;2018.

40. Li J, Jin Q, Huang F, Tang Z and Huang J. Effects of Rab27A and Rab27B on Invasion, Proliferation, Apoptosis, and Chemoresistance in Human Pancreatic Cancer Cells.Pancreas.2017;46:1173-1179.

\section{Figures}



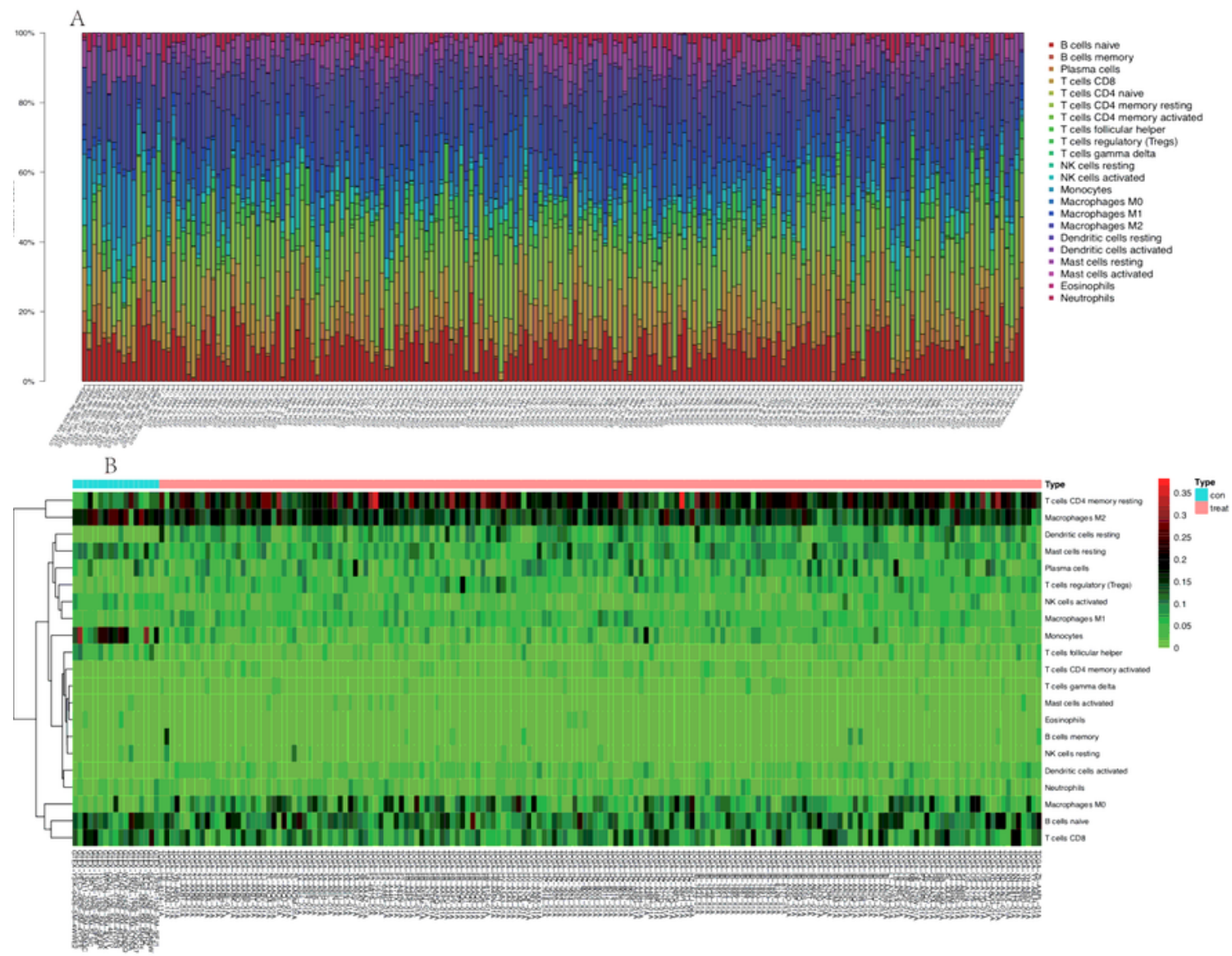

C

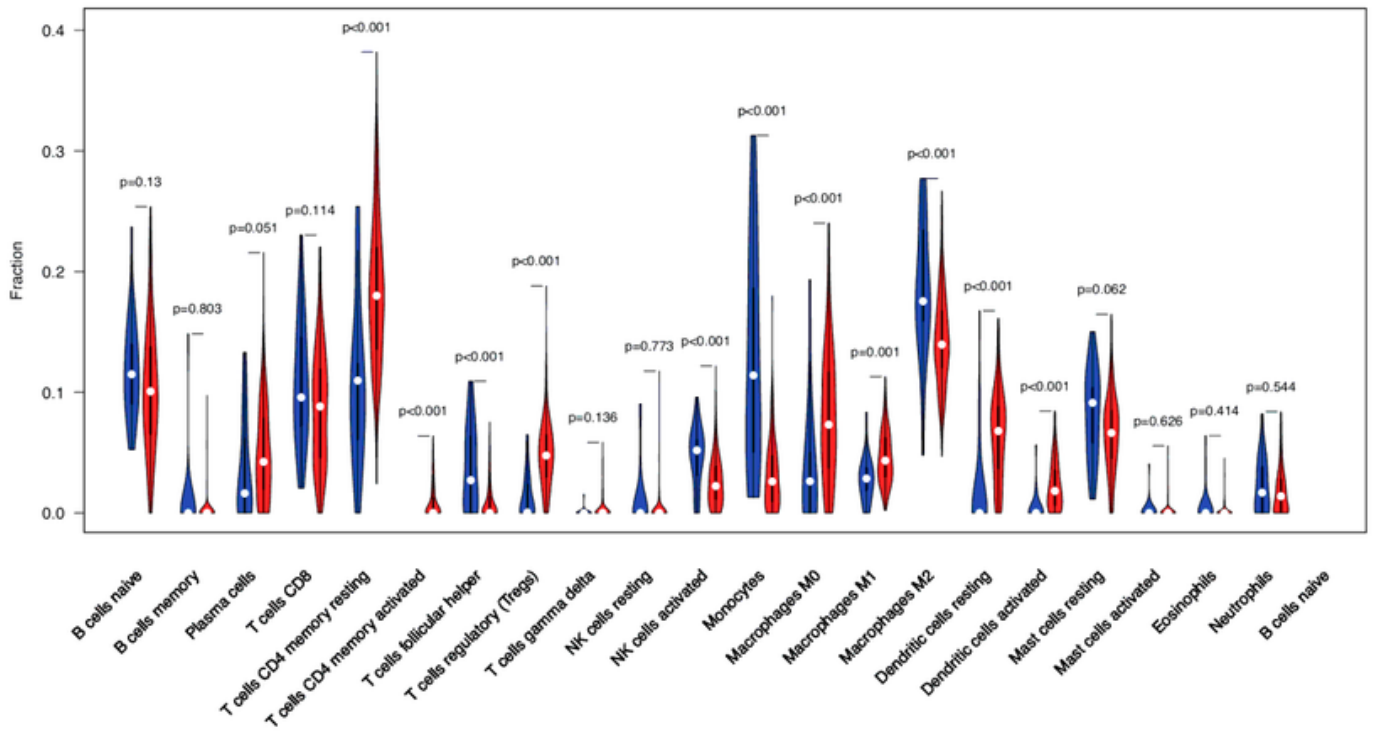

Figure 1

Compositions of immune cells in PC.(a)Bar chart of immune cells composition in pancreatic cancer and normal pancreas.(b)Heatmap of immune cells composition in pancreatic cancer and normal pancreas. (c)Violin chart depicting the difference of immune cells and CIBERSORT p-value. 

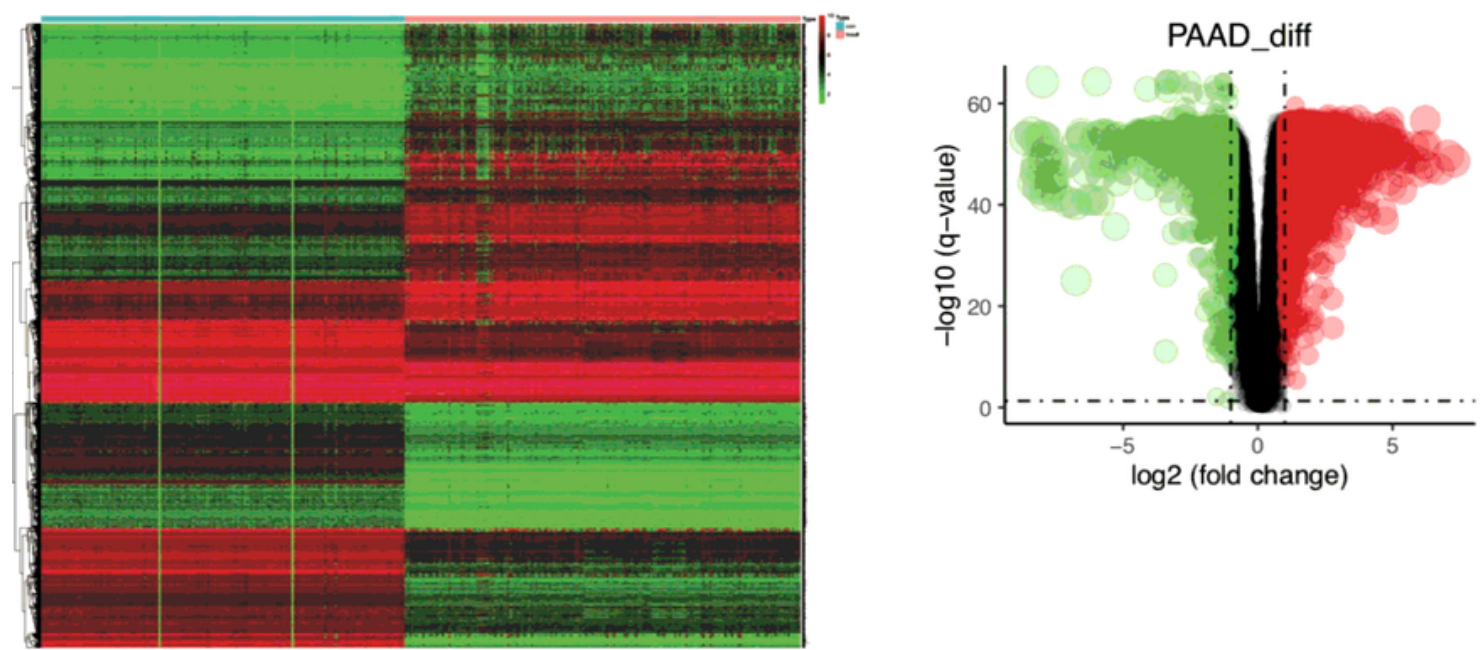

C

$\mathrm{D}$
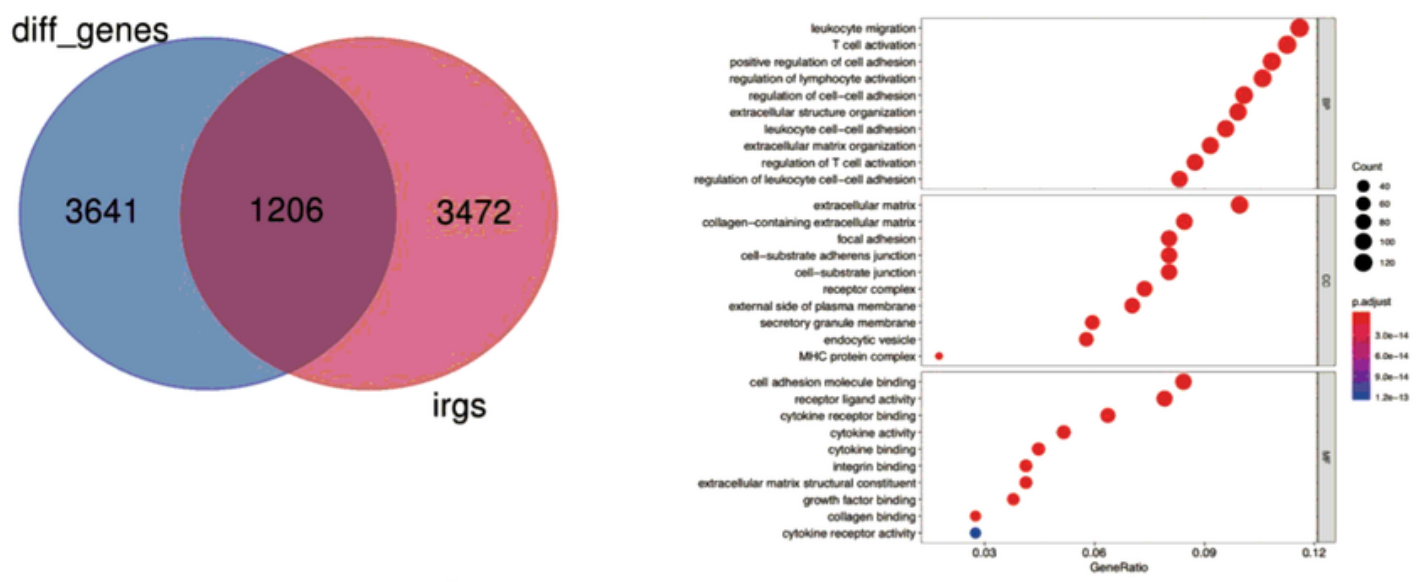

E

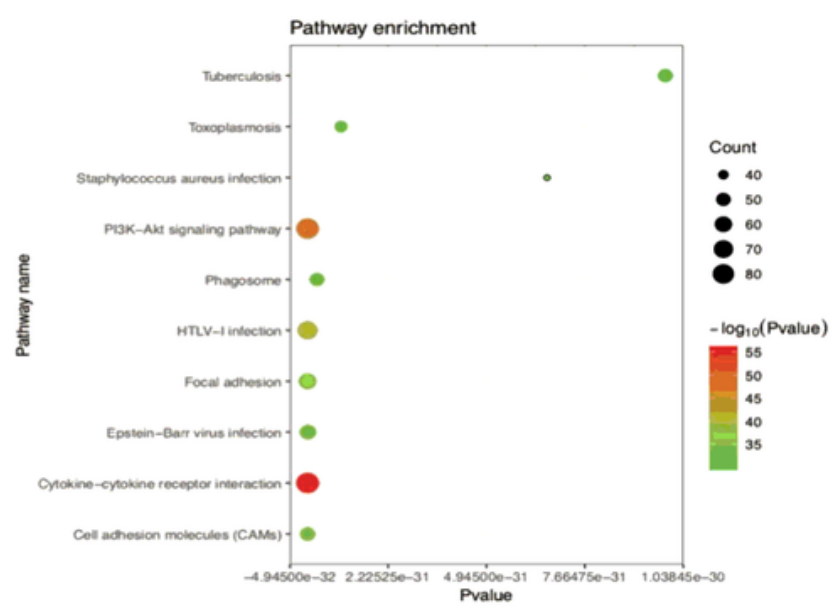

\section{Figure 2}

The identification of differentially expressed genes (DEG).(a) Heatmap demonstrating differentially expressed genes between pancreatic cancer(PC) and non-tumor tissues.(b) Volcano plot demonstrating differentially expressed genes between pancreatic cancer(PC) and non-tumor tissues.(c)Venn plot demonstrating the intersection of differentially expressed genes and immune related genes.Gene functional enrichment of differentially expressed immune-related genes.(d) Gene ontology analysis of 
;biological process, cellular component and molecular function, respectively.(e) The top 10 most significant Kyoto Encyclopedia of Genes and Genomes pathways.

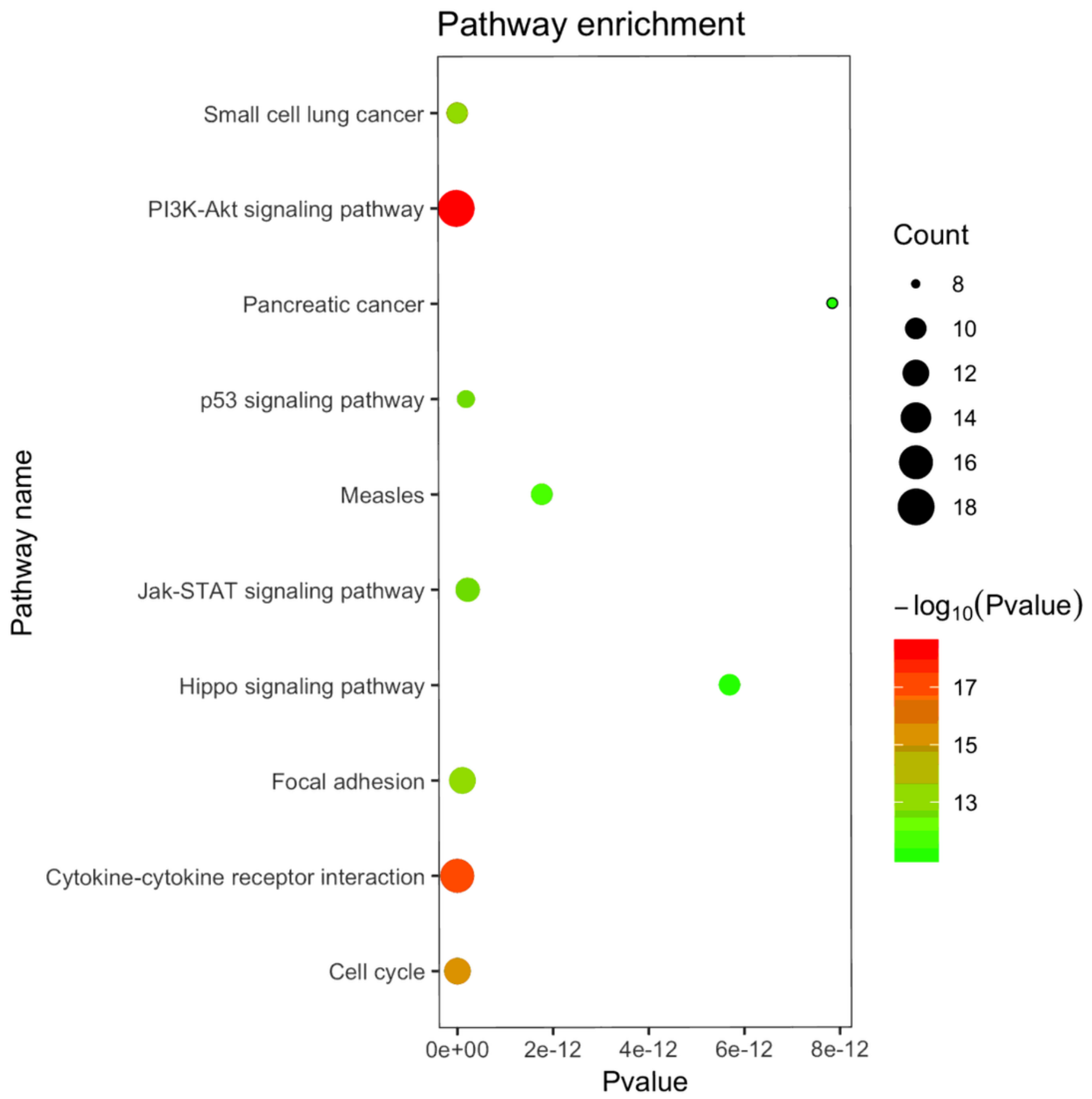

\section{Figure 3}

The identification of OS-related IRGs. The top 10 most significant KEGG pathways. 

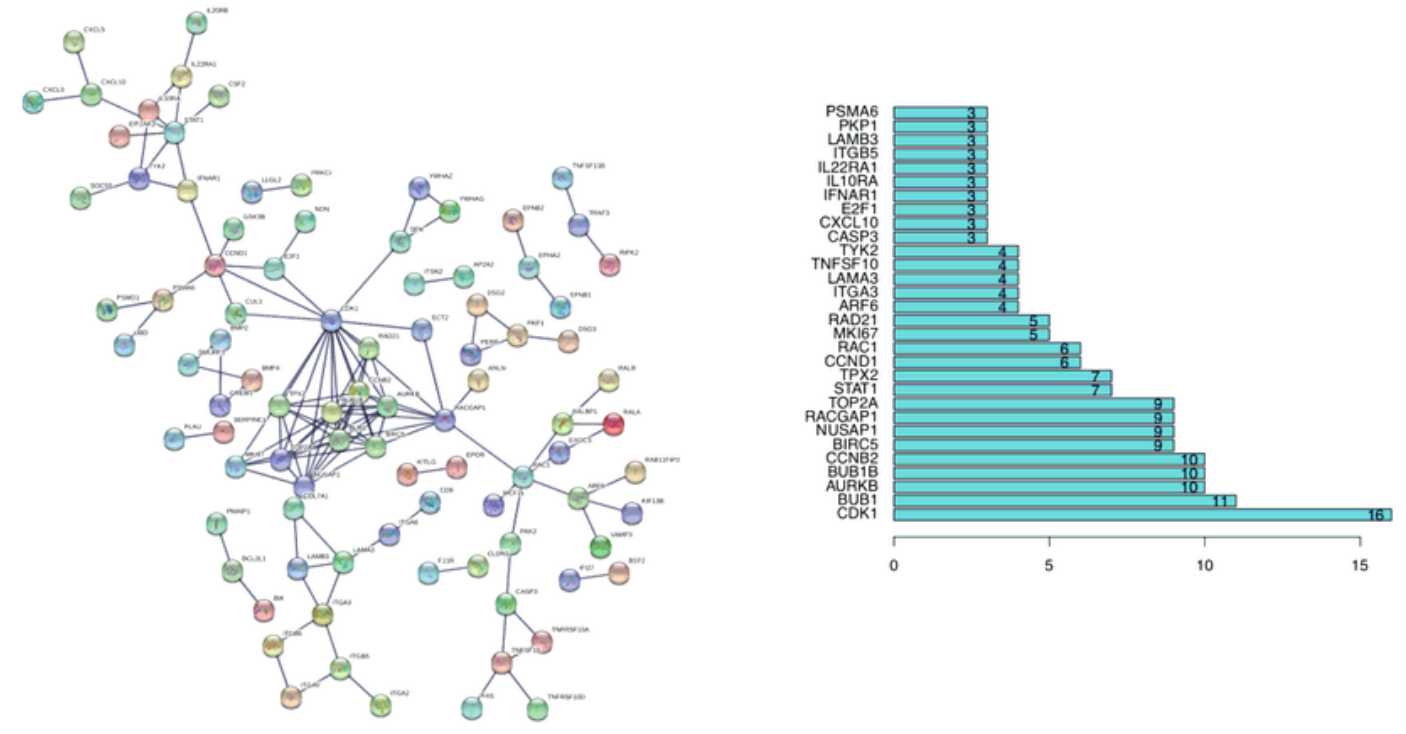

C

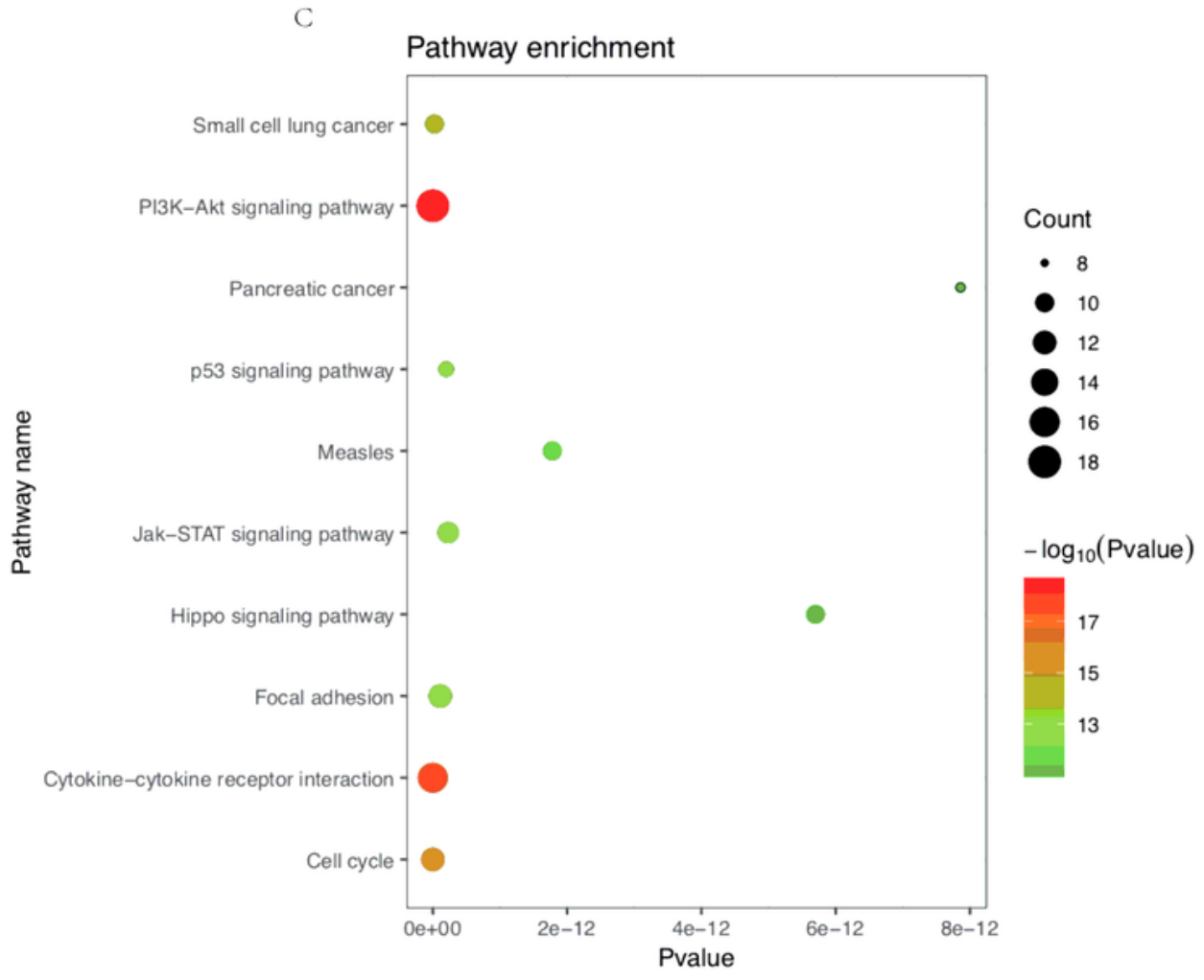

\section{Figure 4}

The identification of hub IRGs .(a) The protein-protein interaction (PPI) network of OS-related IRGs.(b) Barplot of hub genes in PPI.(c) The top 10 most significant KEGG pathways. 
A
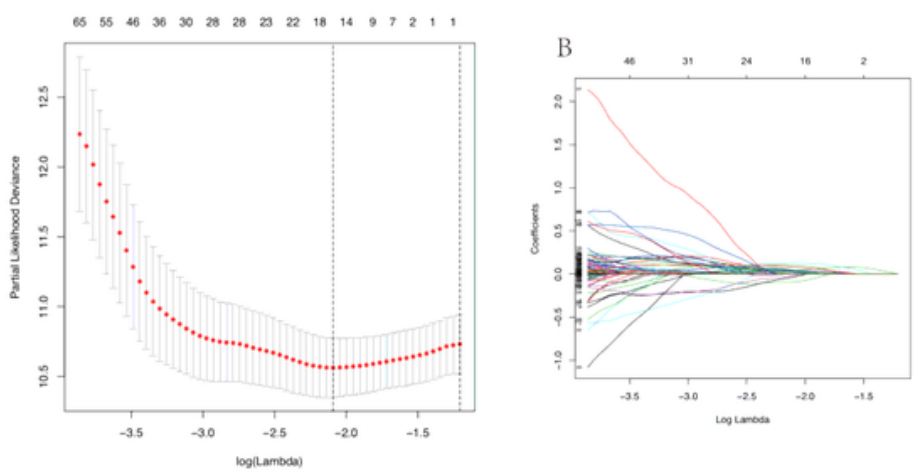

C

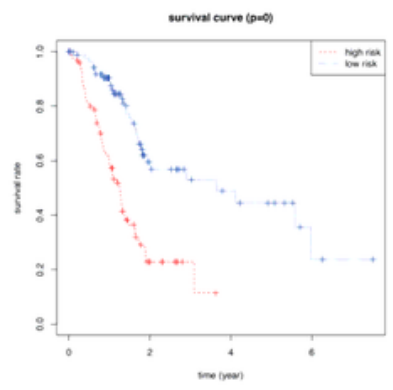

$\mathrm{D}$
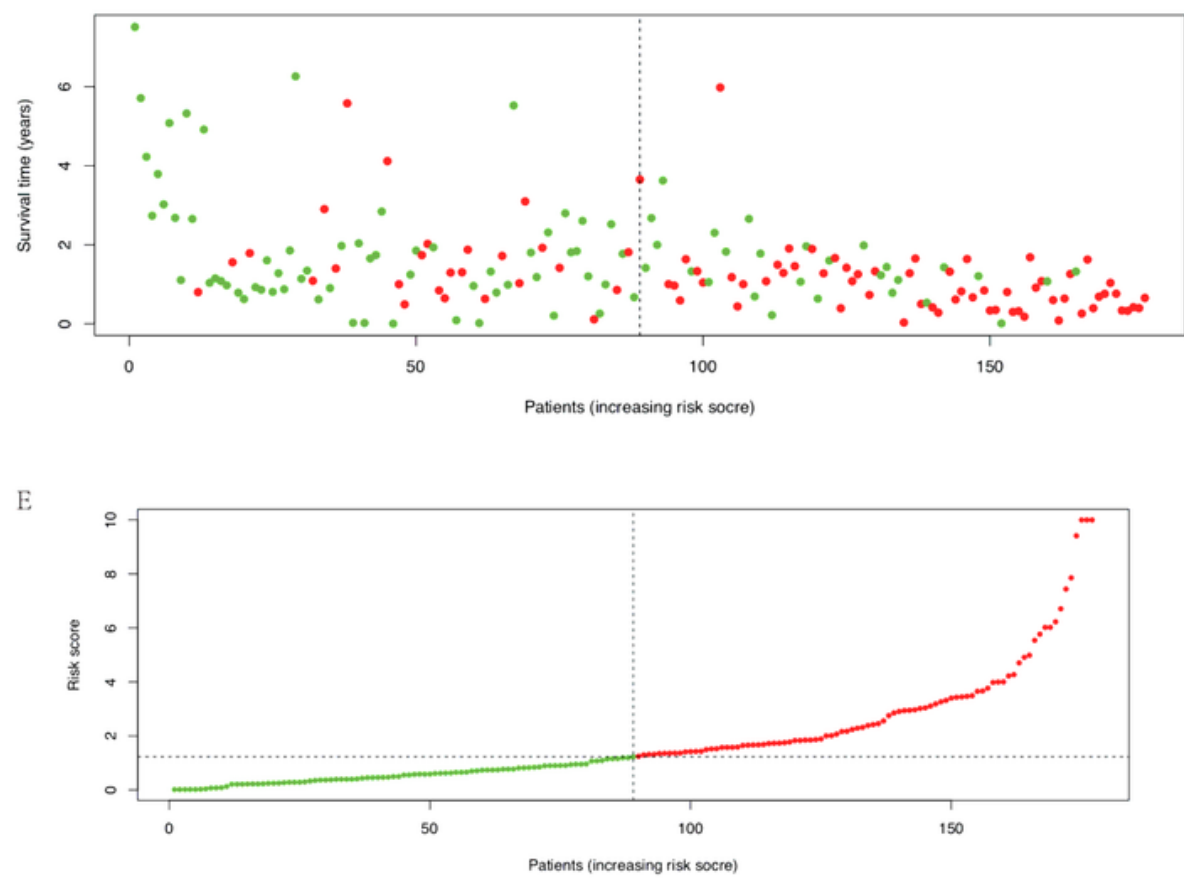

F

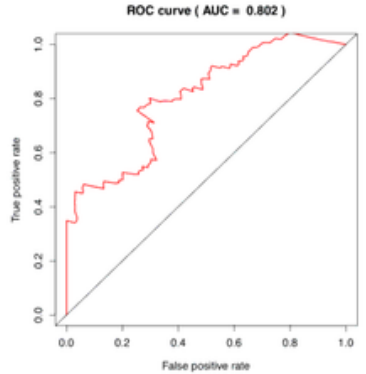

Figure 5

Assessment of clinical outcomes.(a-b) LASSO regression model of OS-related IRGs.(c)Kaplan-Meier analysis of overall survival of patients in different group.(d) Survival status of patients in different groups.(e)Rank of prognostic index and distribution of groups.(f) Receiver operating characteristic curve of IRGs. 

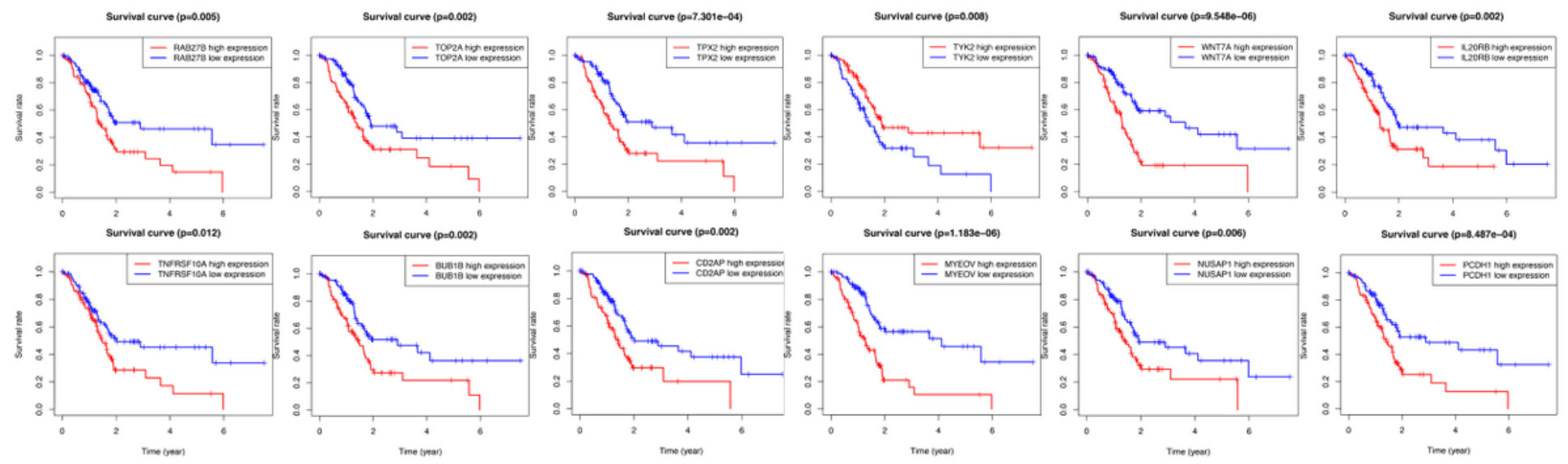

\section{Figure 6}

Kaplan-Meier analysis of overall survival of patients stratified by the expression of 12 selected key IRGs.

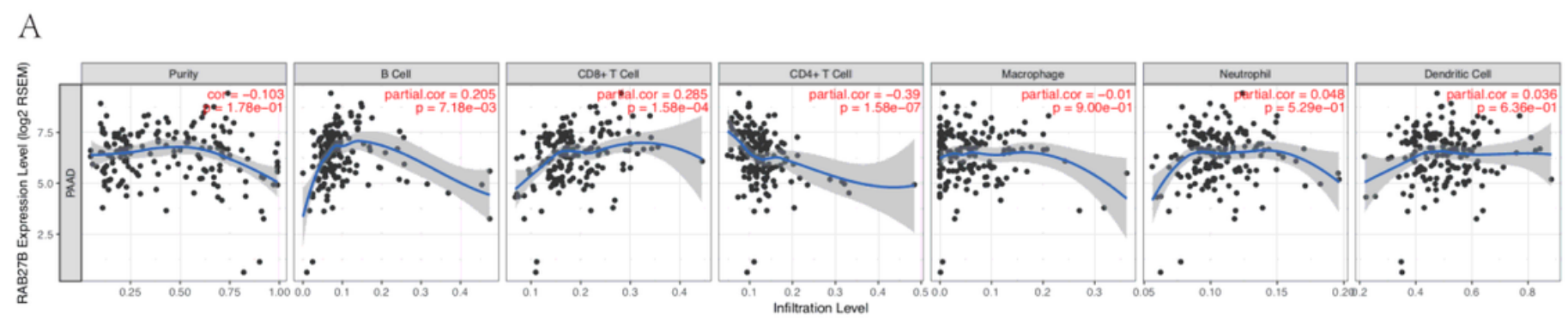

B
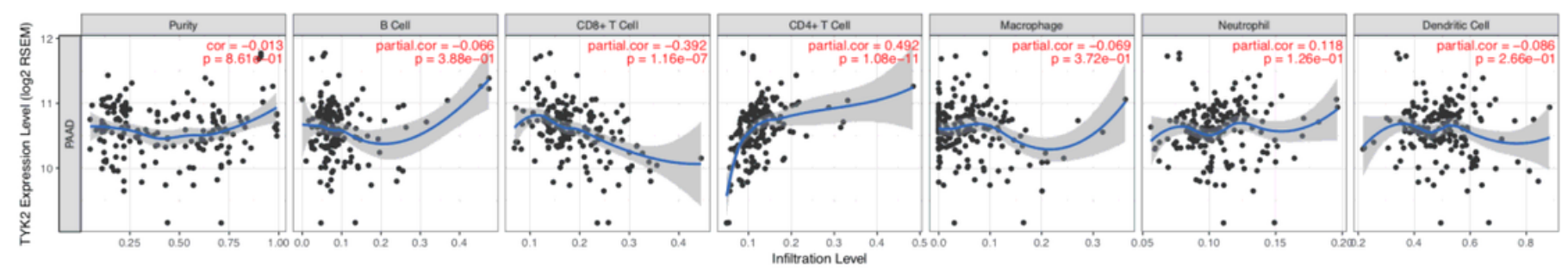

Figure 7

Pearson correlation between key IRGs and immune cells Infiltrating.(a) Pearson correlation between RAB27B and immune cells Infiltrating.(b) Pearson correlation between TYK2 and immune cells Infiltrating 